|| ISSN(online): 2589-8698 || ISSN(print): 2589-868X ||

International Journal of Medical and Biomedical Studies

Available Online at www.ijmbs.info

NLM (National Library of Medicine ID: 101738825)

Index Copernicus Value 2019: 79.34

Original Research Article

Volume 5, Issue 11; November: 2021; Page No. 23-31

\title{
A COMPARATIVE STUDY OF FETAL ADRENAL GLAND BIOMETRY AND CERVICAL LENGTH AS A PREDICTOR OF PRETERM BIRTH
}

\author{
${ }^{1}$ Dr Toshi Jain, ${ }^{2}$ Dr Megha Agrawal, ${ }^{3}$ Dr Anju Sharma \\ ${ }^{1}$ Resident, ${ }^{2}$ Associate Professor, ${ }^{3}$ Senior Professor, \\ Department of Obstetrics and Gynecology, SMS Medical College, Jaipur
}

Article Info: Received 04 October 2021; Accepted 07 November 2021

DOI: https://doi.org/10.32553/ijmbs.v5i11.2282

Corresponding author: Dr Toshi Jain

Conflict of interest: No conflict of interest.

\section{Abstract}

Background: Preterm birth is an important challenge in obstetrics and contemporary perinatology in India. Timely recognition, intervention and appropriate management is integral in curbing the upsurge in its incidence and consequent poor perinatal outcome. This study was conducted taking into account the potential mechanism of preterm labor: premature activation of the placental-adrenal endocrine axis wherein elevation of maternal cortisol leads to an increased production of placental corticotrophin releasing hormone which causes an increase in dehydroepiandrosterone produced by the fetal zone of the adrenal gland and its enlargement. This activates a cascade leading to early loss of uterine quiescence, consequently causing cervical modelling, ripening and preterm birth.

Aim and Objectives: To assess fetal adrenal gland volume, fetal zone enlargement and cervical length on ultrasound and compare their efficacy in the prediction of preterm birth.

Methods: This was a hospital based prospective study in which pregnant women with an uncomplicated live singleton pregnancy between 28 to 34 weeks of gestation were subjected to obstetric ultrasonography wherein fetal adrenal gland volume, fetal zone enlargement and cervical length was measured. They were then followed up until their delivery, whether term or preterm and its correlation with fetal adrenal gland parameters and cervical length was assessed.

Result: Corrected fetal adrenal gland volume showed the highest sensitivity of $90.0 \%$ and a specificity of $96.7 \%$ with a cut off value of $632.50 \mathrm{~mm} 3 / \mathrm{kg}$ while fetal zone enlargement showed a sensitivity and specificity of $72.7 \%$ and $60.9 \%$ respectively. Cervical length was found to be the least important marker for predicting the preterm birth as having the least AUC as 0.209, sensitivity as $36.4 \%$ and specificity as $76.1 \%$.

Conclusion: This study concludes that fetal adrenal gland biometry can be used as a noninvasive, cost effective and potential new marker for the prediction of preterm birth and is a better predictor than cervical length.

Keywords: Preterm, Cervical length, Adrenal biometry.

\section{Introduction}

Preterm birth is defined as the onset of delivery prior to 37 completed weeks but after the age of viability, which may range from 24 weeks to 28 weeks, based on which those born before 28 weeks are termed as extreme preterm and those above 32 weeks are late preterm ${ }^{1}$.

Preterm labor remains to be one of the most important challenges of contemporary perinatology and constitutes one of the main reasons of perinatal mortality. Every year an estimated 15 billion babies are born preterm. More than 60 percent of these preterm births occur in Asia and Africa $^{2}$. Preterm birth is the leading cause of death among children less than five years of age, constituting to about one million deaths every year.

India contributes the greatest number of preterm births worldwide. Out of 27 million babies born every year in
India, 3.5 million babies are born preterm ${ }^{2}$. Out of these, almost three fourths of mortalities could be prevented by cost-effective interventions. Due to the upsurge in the incidence of preterm labor and increased annual cost in the care of preterm infants, there is a definite need to find a highly sensitive and specific, noninvasive, cost effective and quantitative marker for the prediction of preterm labor for its timely management to reduce the consequent neonatal morbidity and mortality. The potential mechanism for preterm births is the premature activation of the placental-adrenal endocrine axis. Elevation of maternal cortisol activates this axis, yielding the increased production of placental corticotrophin releasing hormone. This causes an increase in dehydroepiandrosterone produced by the fetal zone of the adrenal gland. This activates a cascade leading to an early loss of uterine 
quiescence. The end result of this pathway is cervical modelling and ripening. Due to this physiological chain of events, there is a consequent enlargement of the fetal adrenal gland. Measurement of the fetal adrenal gland volume and the fetal zone enlargement can be used as a predictor of preterm labor. This study was performed in a tertiary care hospital in the state of Rajasthan. Rural population makes up the majority of the patients we cater to, wherein antenatal visits early in pregnancy are a rare sight. Most of these women attend the hospital in their third trimester of pregnancy for the very first time. Thus, this study aims to analyze the role of fetal adrenal gland biometry as a predictor of preterm birth in women with 28 weeks of gestation to 34 weeks of gestation attending the antenatal clinic. This would aid in the prediction of preterm labor in women presenting late in pregnancy and effective interventions and treatment to improve the perinatal outcome. A very few studies have been done in India about the role of fetal adrenal gland biometry in preterm birth. This study aims to evaluate fetal adrenal gland biometry and consolidate the existing literature on its role in the prediction of preterm labor and also compare its efficacy with cervical length assessment.

\section{Aims and Objectives}

The aim of this study is to assess fetal adrenal gland volume,fetal zone enlargement and cervical length on ultrasound and compare their efficacy in the prediction of preterm birth.

\section{Material and Methods}

This was a hospital based prospective cohort study conducted in the department of Obstetrics and Gynecology in SMS Medical College, Jaipur wherein 103 pregnant women with an uncomplicated singleton live pregnancy of 28-34 weeks of gestation fulfilling the inclusion criteria and giving a written, informed consent were recruited for the study. Sample size of 120 pregnant women was taken for the present study which was calculated at $80 \%$ study power and an alpha error of 0.05, assuming an AUC of 0.569 for cervical length in predicting preterm labor and $50 \%$ incidence of preterm labor in the cohort as per results of seed article, expecting $20 \%$ dropouts or loss to follow up or attrition. The final sample size was 103 participants as seventeen patients were lost to follow up due to the COVID 19 pandemic. Patients with an uncomplicated singleton live pregnancy with 28 to 34 weeks of gestational age, willing to participate in the study, with or without prior preterm births or abortions were included in the study. Patients having multifetal pregnancy, polyhydramnios, pregnancy with complications like antepartum hemorrhage, preeclampsia and pregnancy with medical illness like diabetes mellitus, heart disease, fetal abnormalities incompatible with life, uterine anomalies, Urinary tract infection and other bacterial infections were excluded from the study.Gestational age was determined from the last menstrual period and a first trimester scan. Approval from the institutional research, review board and ethical committee was taken.Standardized data collection on a predesigned study proforma including a full antenatal workup and routine investigations was done. This included detailed history and examination of the patient and panel of all routine investigations.Patients were subjected to obstetric ultrasonography wherein fetal adrenal gland biometry and cervical length were assessed. Ultrasound was done using linear array transducer of 9-11 $\mathrm{MHz}$ frequency and a linear obstetrics preset. Fetal adrenals can be focussed in the axial plane, by smoothly navigating the transducer cranially above the level of kidneys. The central hyperechoic part of the fetal adrenal gland (fetal zone) is measured in the sagittal or coronal plane along with the maximum dimension of the gland in the same plane to give fetal zone depth (d) and total gland depth (D).

The $d / D$ ratio is charted as a percentage and called as a marker of fetal zone enlargement (FZE) The total gland dimensions are taken in three orthogonal planes and gland volume is estimated using the ellipsoid formula $\left(a^{*} b^{*} c^{*} 0.52\right)$. This is further divided by the estimated fetal weight (EFW) in kilograms to yield corrected fetal adrenal gland volume (cAGV) in cubic millimeters per kilograms $(\mathrm{mm} 3 / \mathrm{kg})$. EFW is calculated automatically using Hadlock formula based on four common fetal measurements i.e. biparietal diameter (BPD), head circumference (HC), abdominal circumference (AC), and femur length (FL).

These measurements were done on both adrenal glands however, left-sided measurements were used due to its semilunar shape. In patients with increased abdominal fat, the fetal adrenals were assessed using a $3.5 \mathrm{MHz}$ curvilinear probe. All these women were followed up until delivery, whether preterm or term and its correlation with the fetal adrenal gland parameters were assessed.

\section{Result}

The mean age of women who delivered preterm was found to be $25.63 \pm 4.20$ years and the mean age of women who delivered at term was also found to be $26.04 \pm 4.05$ years. 54.54 percent were lying in the age group of $20-25$ in both preterm and term group. 36.36 percent in preterm group and 31.52 percent in term were lying in the age group of 26-30. 9.78 percent in preterm group and 9.09 percent in term group of 31-35 age group. 4.34 percent were found to be in age group of 36-40 in term group. On comparing it was found that age of the two groups in the study was comparable $(\mathrm{p}=0.99)$. 


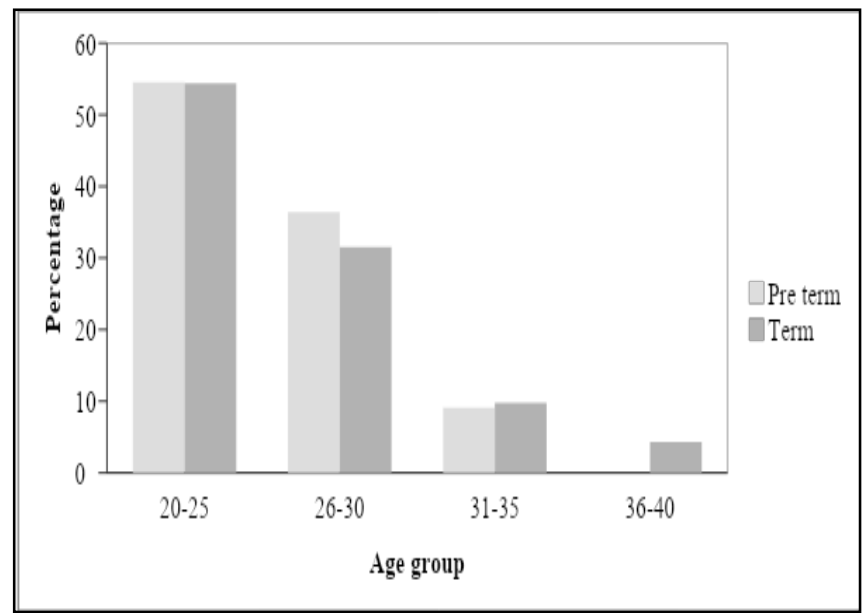

Figure 1: Distribution of women with respect to age

Table 1: Age distribution of patients

\begin{tabular}{|l|c|c|c|c|c|}
\hline Age & Preterm(11) & Percentage & Term(92) & Percentage & P value \\
\hline $20-25$ & 6 & 54.54 & 50 & 54.35 & 0.99 \\
\cline { 1 - 5 } $26-30$ & 4 & 36.36 & 29 & 31.52 & \\
\cline { 1 - 5 } $31-35$ & 1 & 9.09 & 9 & 9.78 \\
\hline $36-40$ & 0 & 0 & 4 & 4.34 & \\
\hline Total & 11 & 100 & 92 & 100 & \\
\cline { 1 - 5 }
\end{tabular}

This table concludes that out of 11 patients who delivered preterm, 10 were primigravida while only one patient was second gravida. Out of the 92 women who delivered at term, 55 were primigravida, 25 were G2 and 14 were G3.

The gravidity of patients who delivered preterm and who delivered at term have no significant difference as p value is 0.9

Table 2: Distribution of the participants in terms of gravidity

\begin{tabular}{|l|c|l|l|l|l|}
\hline Variable & Preterm(11) & Percentage & Term(92) & Percentage & P value \\
\hline G1 & 10 & 90.90 & 55 & 59.78 & 0.99 \\
\hline G2 & 1 & 9.09 & 23 & 25.00 & \\
\hline TG3 & 0 & 0 & 14 & 15.22 & \\
\hline Total & $\mathbf{1 1}$ & 100 & 92 & 100 & \\
\hline
\end{tabular}

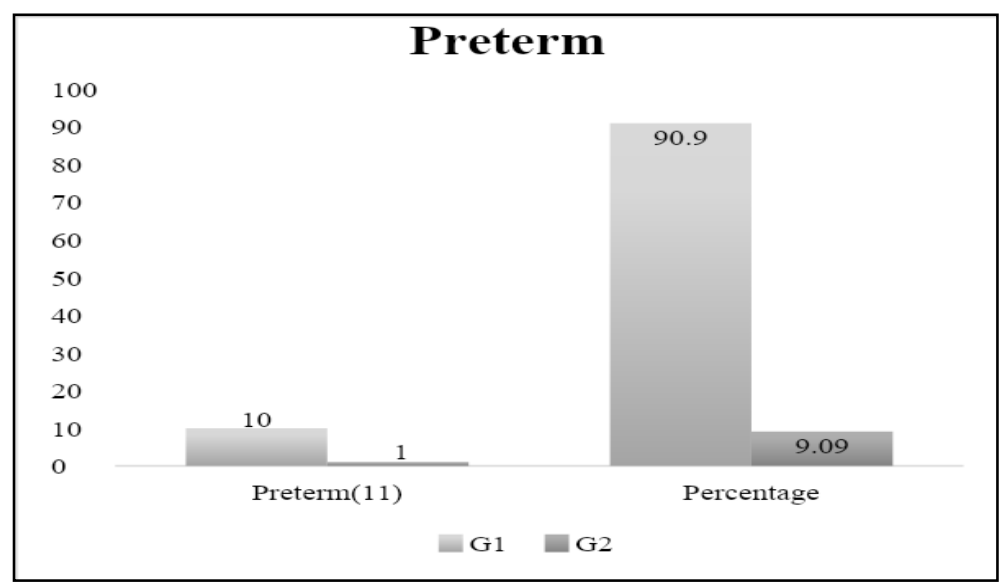




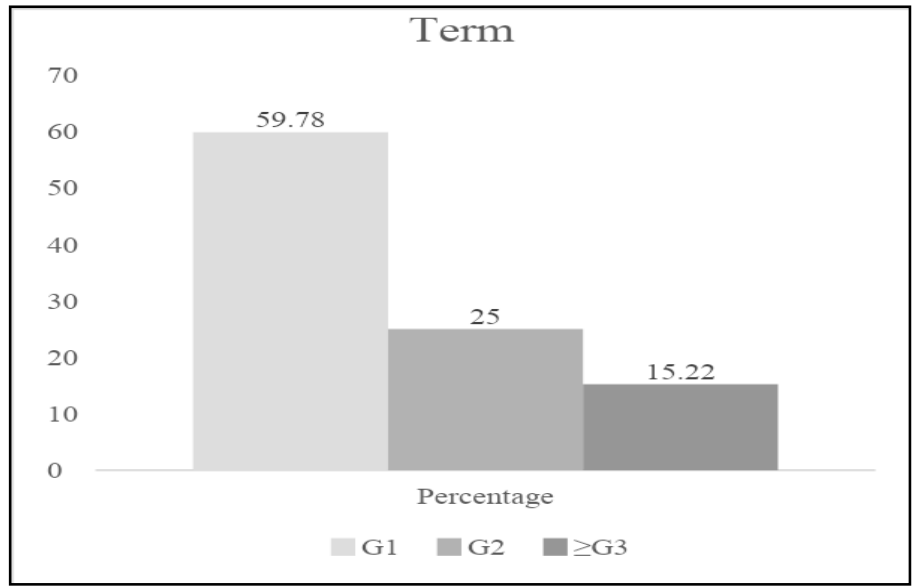

Figure 2: Distribution of patients with respect to gravidity

The gestational age of term delivery group was found to be $32.13 \pm 2.09$ months and for term delivery group it was found to be $31.79 \pm 1.88$ months at the time of ultrasound examination.. There was no significant difference found in the gestational age at time of ultrasound as the $p$ value was calculated as 0.611 .
The gestational age of patients who delivered preterm was found to be $35.36 \pm 0.92$ months and patients who delivered at term was found to be $38.56 \pm 0.61$ months. There was significant difference found in the gestational age at time of delivery for both the groups as the $\mathrm{p}$ value was calculated as 0.001 .

Table 3: Gestational age at Time of Delivery

\begin{tabular}{|c|c|c|c|}
\hline Variable & Preterm(11) & $\operatorname{Term}(92)$ & P value \\
\hline$<37$ weeks & 11 & 0 & \multirow[t]{3}{*}{0.001} \\
\hline$>37$ weeks & 0 & 92 & \\
\hline Mean \pm SD & $35.36364 \pm 0.924416$ & $38.56522 \pm 0.616697$ & \\
\hline
\end{tabular}

Table 4: Descriptive statistics

\begin{tabular}{|l|l|l|l|}
\hline Variable & Preterm(11) & Term(92) & P value \\
\hline Gravidity ,median(range) & $1(1-2)$ & $1(1-4)$ & NA \\
\hline History of Preterm n(\%) & $1(9.09)$ & $1(1.075)$ & NA \\
\hline Output & & & \\
\hline cervical length(SD)(mm) & $33(5.138093)$ & $37.51087(4.693916)$ & 0.016 \\
\hline Estimated Fetal weight(SD)(kg) & $1.8745455(0.4029979)$ & $2.7757143(0.3068128)$ & 0.521 \\
\hline Fetal Zone Depth(SD) & $11.972727(3.970917)$ & $8.5978261(2.2640515)$ & 0.0186719 \\
\hline Fetal zone enlargement, \%, mean (SD) & $0.61827(0.1226673)$ & $0.5358444(0.0587409)$ & 0.0235 \\
\hline Corrected adrenal gland volume,mm3/kg, mean (SD) & $769.07091(85.343434)$ & $280.5645(38.830805)$ & 0.001 \\
\hline
\end{tabular}

The ultrasound measures of both groups of patients are shown in Table 4.Mean values of the continuous variables like cervical length, estimated fetal weight, fetal zone depth, fetal zone enlargement and corrected adrenal gland volume were tabulated as outcome measures.

The mean fetal weight in women who delivered preterm was $1.87 \mathrm{~kg}$ while in those who delivered at term was 2.77 $\mathrm{kg}$ with a $\mathrm{p}$ value of 0.521 .

The mean cervical length in women who delivered preterm was $33 \mathrm{~mm}$ and in those who delivered at term was 37.5 $\mathrm{mm}$. The cervical length was found to be significantly lower for preterm group in comparison to term group. The $\mathrm{p}$ value for the same was 0.016 which makes it statistically significant.

The test revealed that preterm pregnant women had statistically significant greater fetal adrenal volume and fetal zone depth than those delivered at term. The mean (SD)corrected fetal adrenal gland volume for women who delivered preterm was $769.07 \mathrm{~mm} 3 / \mathrm{kg}$ and that in the women who delivered at term was $280.564 \mathrm{~mm} 3 / \mathrm{kg}$. The $\mathrm{p}$ value was calculated as 0.001 which is statistically significant.

The mean fetal zone depth in patients who delivered preterm was $11.97 \mathrm{~mm}$ while in those who delivered at 
term was 8.59 . The $\mathrm{p}$ value was 0.018 which is statistically significant. Mean fetal zone enlargement in patients who delivered preterm was 0.618 whereas it was 0.535 in patients who delivered at term. The $\mathrm{p}$ value calculated was 0.0235 which is statistically significant.
The markers of fetal adrenal gland enlargement i.e. FZE and $\mathrm{cAGV}$ showed higher values in the women who delivered preterm than term and that too with statistical significance.

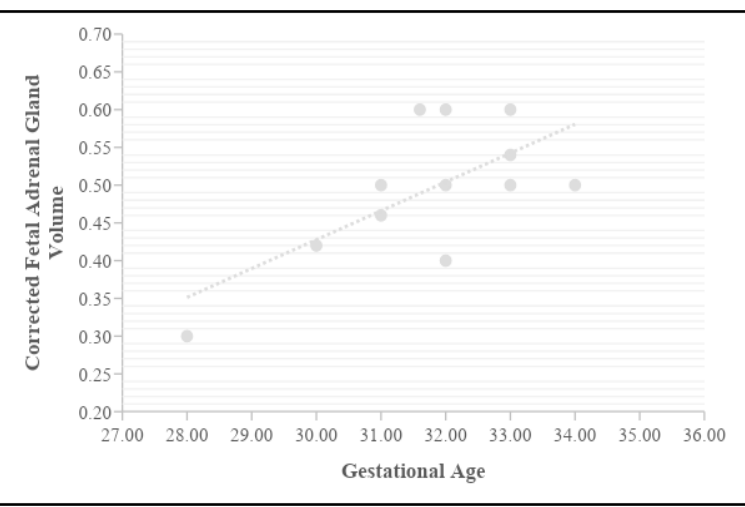

Figure 3: Linear regression graph between corrected adrenal gland volume and gestational age

This is a linear regression graph between corrected fetal adrenal gland volume (cAGV) and gestational age. As it can be seen in the scattered plot, there is a positive correlation between gestational age and corrected fetal adrenal gland volume however it is a weak correlation.

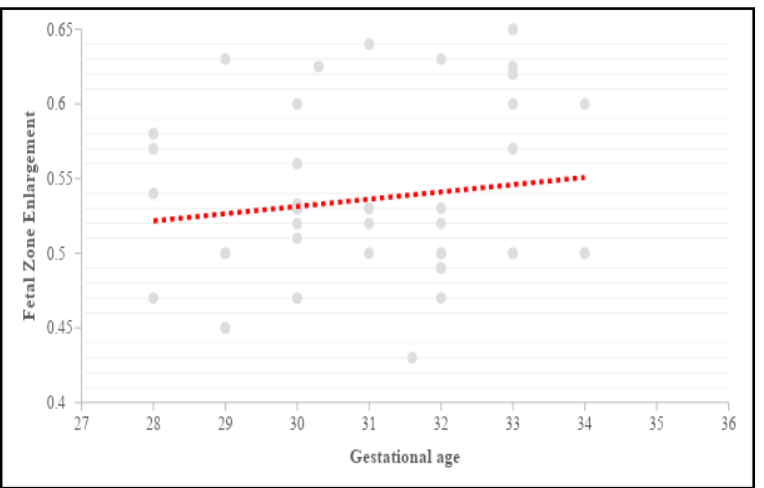

Figure 4: Linear regression graph between fetal zone enlargement and gestational age

This is a linear regression graph between fetal zone enlargement and gestational age. As can be seen from the graph, there is no significant correlation between both these variables.

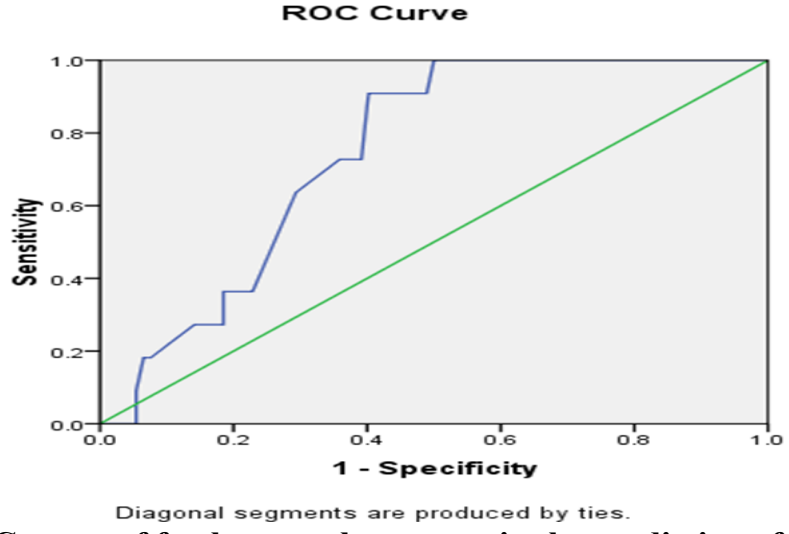

Figure 5: ROC curve of fetal zone enlargement in the prediction of preterm birth 


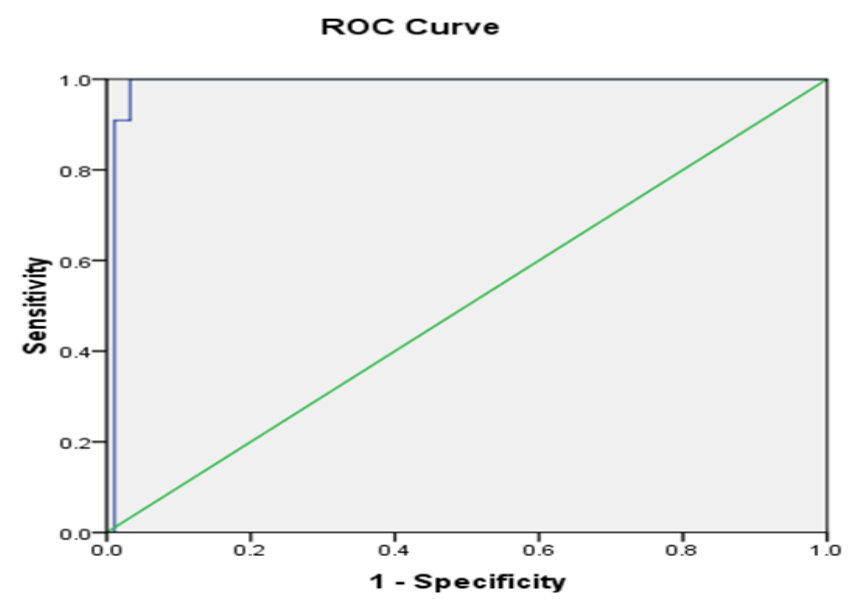

Figure 6: ROC curve of corrected adrenal gland volume in the prediction of preterm birth

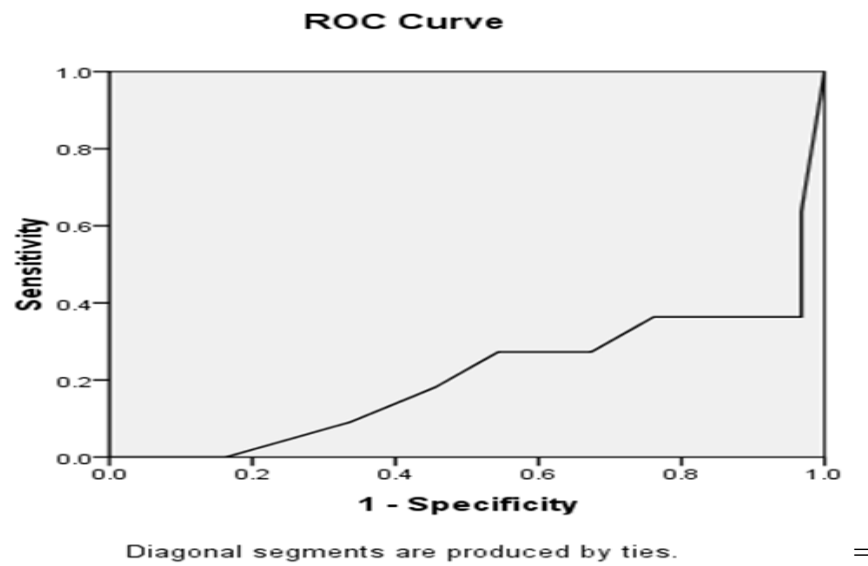

Figure 7: ROC curve of cervical length in the prediction of preterm birth

Table 5: Sensitivity and specificity of test variables at their ROC curve derived cut off values

\begin{tabular}{|l|l|l|l|l|}
\hline & AUC & Cut off value & Sensitivity & Specificity \\
\hline Cervical Length & 20.9 & 35.5 & 36.4 & 76.1 \\
\hline Fetal zone enlargement & 74.5 & 58.50 & 72.7 & 60.9 \\
\hline CAGV & 98.7 & 632.50 & 90.9 & 96.7 \\
\hline
\end{tabular}

ROC curves were plotted with Table 5 showing cut-off values of variables (derived from ROC curves) and their corresponding sensitivities and specificities. $\mathrm{cAGV}$ at the cut-off value of $632.50 \mathrm{~mm} 3 / \mathrm{kg}$ showed the highest area under the curve, AUC (0.987), sensitivity (90.9\%), and specificity $(96.7 \%)$ for prediction of preterm birth. FZE also showed a significant AUC as 0.745 , sensitivity as $72.7 \%$ and specificity as $60.9 \%$. Cervical length was found to be the least important marker for predicting the preterm birth as having the least AUC as 0.209 , sensitivity as 36.4 $\%$ and specificity as $76.1 \%$.

Once supported by more comprehensive studies, this result could be beneficial in the prediction of preterm labor in clinical practice.

\section{Discussion}

This study was done in the department of Obstetrics and Gynaecology in SMS Medical College, Jaipur. Pregnant women with 28-34 weeks of gestation with an uncomplicated singleton live pregnancy with or without history of prior preterm births or abortions were included in the study and subjected to ultrasonography wherein fetal adrenal gland biometry including fetal adrenal gland volume and fetal zone enlargement was assessed and cervical length was also measured. These women were followed up till delivery and delivery outcome in the form of preterm or term delivery and their association with these ultrasonographic parameters were assessed. 
In our study, out of 103 participants, 11 participants delivered preterm and 92 delivered at term. There was no significant difference between the two groups in terms of distribution of age. Our observation was consistent with the study of Theresia B Temu et al $(2016)^{3}$ where maternal age was not associated with increased risk of preterm delivery. This is also consistent with the study of Anand Ahankari et al $(2017)^{4}$ where in they analyzed 655 live births, of which $6.1 \%$ were preterm deliveries. The odds of both preterm delivery and LBW were reduced in multigravida compared with primigravida women regardless of age.

As our hospital caters to majority of the rural population, 91 out of 103 participants had a rural background. This is consistent with the results of Theresia B Temu et al (2016) studies which state that preterm birth is less likely in women who reside in urban areas. More importantly, it concluded that women seeking inadequate antenatal care are more likely to have preterm births which is also evident from this study. This is also consistent with the study of Maria do Carmo Leal et al (2016) ${ }^{5}$ who in their study identified socio-demographic factors associated with spontaneous preterm birth. These were adolescent pregnancy, low total years of schooling, and inadequate prenatal care. Other risk factors were previous preterm birth, multiple pregnancy, abruptio placentae and infections.

The study shows gestational age at the time of ultrasound which shows that patients who delivered preterm had a mean gestation of 32.13 weeks. As mentioned before, being a tertiary care centre catering to a majority of rural population, most of our patients present to the hospital for the first time in their third trimester itself. Thus prediction of preterm birth in patients with no prior antenatal visits becomes very important for timely intervention to reduce the burden of perinatal morbidity and mortality.

The ultrasound examination of patients who delivered preterm and term was done at the same gestational age and there was no significant difference among them. It shows that the examination of fetal adrenal gland biometry and cervical length was done for both the groups at the same gestational age hence the results were able to predict the risk more appropriately and withou any error.

This study shows that patients who delivered preterm had a shorter cervical length than those who delivered at term. The results were coherent with Iams et al.(1996) ${ }^{6}$ who performed secondary analysis on data collected for the Preterm Prediction Study, and found that the rate of cervical shortening was higher in women who went on to have spontaneous preterm birth. Similar results were observed in other studies as well.

This study shows that corrected fetal adrenal gland volume and fetal zone enlargement is significantly higher in patients who delivered preterm than at term. This is consistent with studies done previously viz Turan et al $(2007)^{7}$ prospectively assessed the relationship between fetal adrenal gland volume measurement by 3D ultrasound and preterm birth (birth $<37$ weeks) in 150 women. Sixtyseven women delivered at term and were considered "normal" pregnant controls. Fetal adrenal gland volume and kidney volume changed significantly in a direct linear relationship with estimated fetal weight (fetal adrenal gland volume $r=0.88, P<.001$; kidney volume $r=0.81, P<.001$ ). It was determined that a corrected adrenal gland volume index of more than $422 \mathrm{~mm}^{3} / \mathrm{kg}$ was able to predict preterm birth within 5 days from the date of the scan with the highest accuracy (receiver operating characteristic area: $0.957,95 \%$ confidence interval $0.91-0.99$ ). The sensitivity, specificity, and positive and negative likelihood ratios were $92.0 \%, 99.0 \%, 93.5$, and 0.08 , respectively.

It also concluded that fetal zone enlargement ( $\mathrm{d} / \mathrm{D}$ or FZE) of more than $49.7 \%$ was found to be $100 \%$ sensitive.

Similarly, Shubhra Agarwal, Arjit Agarwal et al (2017) ${ }^{8}$ wherein thirty pregnant females (from 28 to 37 weeks of gestational age), showing clinical signs and delivery outcome of preterm birth were included in the study with an equal number of not-in-labor antenatal females at 37 weeks as controls. Among 30 pregnant females as case and another 30 as controls, fetal zone enlargement showed the sensitivity and specificity ( $90 \%$ and $80 \%$ respectively) in the prediction of preterm birth. Shear wave speed estimation of the antenatal cervix showed the highest sensitivity and specificity ( $96.7 \%$ and $87 \%$ respectively) in the prediction of preterm birth and also showed a strong correlation with fetal adrenal gland enlargement. Fetal adrenal zone enlargement was also shown to be a reliable marker of preterm birth, however, with reduced sensitivity and specificity than shear wave speed. They also found that cAGV was higher in preterm deliveries with $67 \%$ sensitivity and $76 \%$ specificity at cut-off value of 415 $\mathrm{mm} 3 / \mathrm{kg}(\mathrm{p}<0.001)$.

This is also consistent with the study of Chandana S Bhat et al $(2018)^{9}$ wherein among 204 women who developed features of preterm labor eventually had a significantly high cFAGV (404.70 mm3/kg body weight) during the first scan compared to those who reached term asymptomatically (241.35 mm3/ kg body weight). A cutoff value of 271.16 $\mathrm{mm} 3 / \mathrm{kg}$ body weight showed $90 \%$ sensitivity and $81.9 \%$ specificity. Fetal adrenal gland width ratio had the best efficacy (sensitivity $96.67 \%$, specificity $86.2 \%$ ) followed by cFAGV (sensitivity 96.67\%, specificity 83\%) for predicting preterm delivery.

This is also consistent with A C Gimovsky et al (2019) ${ }^{10}$ which was a diagnostic test accuracy study using a prospective cohort design evaluating the ability of 2dimensional ultrasound measurement of fetal adrenal gland total length, total width, fetal zone length and fetal zone width in women in the third trimester to predict the primary 
outcome of spontaneous term labor. Secondary outcomes were vaginal delivery, length of labor, and maternal and neonatal morbidities.

Of 43 patients recruited, 3 were excluded. 11 presented in spontaneous labor and 29 underwent induction of labor. Patient demographics were similar for all included except for admission cervical exam and oxytocin use. A receiver operative curve was created to assess test predictability. Weighted width of fetal adrenal gland was the best predictor of spontaneous labor amongst variables measured with an area under the curve of $0.674, p=0.93$. w/W $\geq 0.41$ had a sensitivity of $91.0 \%$, specificity of $44.8 \%$, positive predictive value of $38.5 \%$ and a negative predictive value of $92.3 \%$. Maternal and neonatal morbidities were not different between the spontaneous labor group and the induction of labor group.It was concluded that ultrasound measured fetal $\mathrm{w} / \mathrm{W}$ was moderately predictive of spontaneous labor.

In the study by $\mathrm{H}$ M Abo Shady et al (2020) ${ }^{11}$ thirty-three preterm pregnant women at 29-36 weeks of gestation with single pregnancy and clinical diagnosis of preterm labor and control group of 33 pregnant women at 37-40 weeks with term pregnancy were included in this study. Fetal Zone Depth and Total Gland Depth (TGD) of fetal adrenal gland were ultrasonographically measured and FZD/TGD ratios were calculated and compared between the two groups. No difference was found between the two groups in respect of age and number of pregnancies $(P>0.05)$. Yet, preterm birth history rupture of membrane, cervical dilatation and mode of delivery were higher in preterm group ( $\mathrm{P}<0.05)$. Fetal adrenal gland FZD/TGD ratio was statistically significantly higher in preterm group compared to the term group $(52 \% \pm 5 \%$ vs. $29 \% \pm 9 \% ; \mathrm{P}<0.001)$. The growth in $\mathrm{FZ}$ as a fetal adaptation mechanism in increased fetal stress in preterm labor cases was at a significant level. This is consistent with this study wherein the mean fetal zone depth in patients who delivered preterm was $11.97 \mathrm{~mm}$ while in those who delivered at term was $8.59 \mathrm{~mm}$. The $\mathrm{p}$ value was 0.018 which is statistically significant. Mean fetal zone enlargement in patients who delivered preterm was 0.618 whereas it was 0.535 in patients who delivered at term. The $\mathrm{p}$ value calculated was 0.0235 which is statistically significant.

This is also coherent with the study by J Goletzke et al $(2020)^{12}$ in which fetal adrenal gland was assessed via transabdominal ultrasound at gestational weeks (gw) 24$26,28-30$, and $34-36$ in a low-risk pregnancy cohort. Longitudinal trajectories of the total gland and the mark (so called fetal zone) as well as ratio of fetal zone width/ total widths $(\mathrm{w} / \mathrm{W})$ were analyzed using repeated ANOVA analyses. To compare trajectories of the ratio $\mathrm{w} / \mathrm{W}$ for preterm and term fetuses respectively, as well as women with and without clinical signs of preterm labor, the propensity score method was applied. Fetal zone width increased over the course of pregnancy $(p<0.0001)$, while the ratio $\mathrm{w} / \mathrm{W}$ decreased $(p<0.0001)(n=327)$. Comparing the trajectories of the ratio $\mathrm{w} / \mathrm{W}$ in fetuses born preterm $(n=11)$ with propensity-score matched term born fetuses $(n=22)$, a decrease between gw 24-26 and 28-30 was observed in both groups, which continued to decrease for the term born fetuses. However, in preterm born fetuses, the ratio increased above the term born values at gestational age 34-36.This study provided longitudinal growth data on the fetal adrenal gland and supports the hypothesis that fetal zone enlargement is associated with preterm birth which could play an important role in risk-prediction.

\section{Conclusion}

The present study demonstrates that preterm delivery is still a challenge in our country. Majority of the risk factors, found to be associated with preterm birth, are modifiable. Modifiable risk factors can be identified and modified as primary prevention strategies. Risk factors like previous preterm birth, multiple pregnancies and placental abnormalities cannot be modified hence preventive efforts should be directed towards modifying working conditions during current pregnancy. Therefore early identification of these factors during prenatal care may prevent the risk of preterm delivery. This calls upon to reinforce antenatal care and services for better birth outcomes. Appropriate and innovative preventive intervention, customized individual needs may prevent preterm births and improve fetal outcomes. Therefore secondary prevention in terms of screening with novel, more sensitive and specific markers like fetal adrenal gland volume and fetal zone enlargement even in the third trimester can aid in reducing the burden of mortality and improve the perinatal outcome in comparison to the established cervical length assessment. Ultrasound being noninvasive, easily reproducible and a cost effective screening method has the potential and feasibility of being of utmost importance to combat preterm birth. Fetal adrenal gland biometry can act as a potential new marker for the prediction of preterm delivery. In order to reach an effective reduction in preterm births, two premises are necessary: to identify women who are at risk by screening with assessment of fetal adrenal biometry and cervical length and to dispose of measures that serve to extend pregnancy by timely interventions and instituting measures.

\section{References}

1. Williams' Obstetrics, $25^{\text {th }}$ edition

2. WHO Preterm birth statistics 2020

3. Theresia B. Temu, Gilead Masenga, Joseph Obure, Dominic Mosha, Michael J. Mahande, "Maternal and obstetric risk factors associated with preterm delivery at a referral hospital in northern -eastern Tanzania." Asian pacific journal of reproduction 2016:5(5):365370. 
4. Anand Ahankari, Sharda Bapat, Puja Myles, Andrew Fogarty, Laila Tata. Factors associated with preterm delivery and low birth weight, 2017,6:72.

5. Maria do Cormo Leal, Ana Paula Esteves-Pereira, Marcos Nakamura-Pereira, Jacqueline Alves Torres, Mariza Theme-Filha, Rosa Maria Soares Madeira Domingues, Marcos Augusto Bastos Dias, Maria Elizabeth Moreira and Silvana Granado Gama, "Prevalence and risk factors related to preterm birth in Brazil.” Reproductive Health 2016 13(suppl 3):127.

6. J. D. Iams, R. L. Goldenberg, P. J. Meis et al., "The Length of the Cervix and the Risk of Spontaneous Premature Delivery," The New England Journal of Medicine, vol. 334, no. 9, pp. 567-573, 1996.

7. Ozhan M Turan, Sifa Turan, Edmund F Funai, Irina A Buhimschi, Joshua A Copel, Catalin S Buhimschi Obstetrics \& Gynecology 109 (4), 855-862, 2007

8. S Agarwal, A Agarwal et al " Fetal adrenal gland biometry and cervical elastography as predictors of preterm birth : a comparative study ", SAGE, Vol. 26(1) 54-62,2018

9. Chandana S Bhat, Sapna Vinit Amin, Prashanth Adiga, Deeksha Pandey The Journal of Obstetrics and Gynecology of India 69 (3), 252-257, 2019

10. Alexis C Gimovsky, Amelie Pham, Philip Shlossman, Matthew Hoffman European Journal of Obstetrics \& Gynecology and Reproductive Biology 240, 341-346, 2019

11. Heba Maged Abo Shady, Mohamed Ahmed Samy Kandil, Ashraf Anas Zytoon, Shaimaa Mahmoud Youssef The Egyptian Journal of Hospital Medicine 84 (1), 1711-1718, 2021

12. Janina Goletzke, Mirja Pagenkemper, Christian Wiessner, Franziska Rüber, Petra Arck, Kurt Hecher, Anke Diemert BMC pregnancy and childbirth 20 (1), $1-9,2020$ 